\title{
Performance Evaluation of Sensor-fused Energy-efficient Geofencing for Smartphones
}

\author{
Tomohiro Nakagawa \\ Research Laboratories, \\ NTT DOCOMO, Inc. \\ 3-6, Hikarino-oka, Yokosuka, \\ Kanagawa, Japan, 239-8536 \\ nakagawatom@nttdocomo.com
}

\author{
Ken Ohta \\ Research Laboratories, \\ NTT DOCOMO, Inc. \\ 3-6, Hikarino-oka, Yokosuka, \\ Kanagawa, Japan, 239-8536 \\ ootaken@nttdocomo.com
}

\author{
Hiroshi Inamura \\ Research Laboratories, \\ NTT DOCOMO, Inc. \\ 3-6, Hikarino-oka, Yokosuka, \\ Kanagawa, Japan, 239-8536 \\ inamura@nttdocomo.com
}

\author{
Makoto Suzuki \\ RCAST, The University of Tokyo. \\ 4-6-1, Komaba, Meguro, \\ Tokyo, Japan, 153-8904 \\ makoto@mlab.t.u-tokyo.ac.jp
}

\author{
Hiroyuki Morikawa \\ RCAST, The University of Tokyo. \\ 4-6-1, Komaba, Meguro, \\ Tokyo, Japan, 153-8904 \\ mori@mlab.t.u-tokyo.ac.jp
}

\begin{abstract}
Geofencing technology enables various location-based applications by detecting entrance to a predefined region and utilizes the event as a trigger to automatic communication or control of smart devices. In this paper, we implement the proposed geofencing system on Android based smartphone and evaluate performance through field trials. The system is composed of movement detection, variable interval positioning, and positioning method switching. We implement both of a system based on the proposed method, and a system based on Android Geofence function. Evaluation results show that the proposed algorithm reduces number of position fix by $79 \%$ compared to Android Geofence function, while maintaining entrance detection delay within acceptable range of 3 minutes.
\end{abstract}

\section{Keywords}

Geofencing, location-based service, Android

\section{INTRODUCTION}

Geofencing technology enables location based services by detecting user's entrance to a predefined region, which is called a target spot, without user's intervention[1][2][3]. For instance, reminder applications can utilize geofencing function to notify the user of arriving at specified station on the way to office or college. As another example, stores which friends with close preference rate high are recommended when these stores are nearby.

By combining various sensors on a smartphone, realtimeness of entrance detection and improvement of power consumption can be supported at the same time. To this end, a geofencing algorithm controls activation and deactivation of an acceleration sensor or positioning sensors.

Permission to make digital or hard copies of all or part of this work for personal or classroom use is granted without fee provided that copies are not made or distributed for profit or commercial advantage and that copies bear this notice and the full citation on the first page. To copy otherwise, to republish, to post on servers or to redistribute to lists, requires prior specific permission and/or a fee.

MOBIQUITOUS 2014, December 02-05, London, Great Britain Copyright (C) 2014 ICST 978-1-63190-039-6

DOI 10.4108/icst.mobiquitous.2014.258038
Based on this approach, we developed an energy-efficient geofencing system and evaluated its performance through computer simulation [4]. In this paper, we implement the system on Android based smartphone and evaluate the performance through field trials.

We classify evaluation scenario into three categories in which system behaves differently according to geofencing algorithms. Even though the user doesn't enter into the target spots, it is required to reduce power consumption. In case the user enters into the target spots, it is still needed to detect the entrance without delay. The scenarios for evaluation covers all those situations.

\section{SENSOR-FUSED GEOFENCING}

Overview of our proposed method is shown in Figure 1. The proposed method is composed of movement detection[5], variable interval positioning[2], and positioning method switching[6].

Movement detection[5] utilizes acceleration sensor to judge if the user is moving, and suppresses power-consuming positioning when the user is stationary. Variable interval positioning[2] adjusts positioning interval in accordance with the distance to target spots. In the proposed method, positioning interval $\mathrm{T}$ is defined as follows:

$$
\mathrm{T}=\max \{\min (\mathrm{d} / \mathrm{Vmax}, \mathrm{Tmax}), \mathrm{Tmin}\}
$$

where $d$ is the distance to the nearest target spot, Vmax is an assumed maximum velocity, and Tmax and Tmin are the maximum and minimum values of $\mathrm{T}$ in order to avoid too long or too short positioning interval.

Positioning method switching[6] activates and deactivates GPS, cell-id based positioning, and WiFi based positioning so that power consumption is reduced when the user is far away from target spots. System parameters for each function are defined by computer simulation (Table. 1).

On the other hand, though detailed algorithm is unknown, Android geofencing function includes variable interval positioning function and positioning method switching. Among the parameters that define the balance between positioning accuracy and power consumption, we chose PRIORITY_NO_POWER, which minimizes power consumption. 


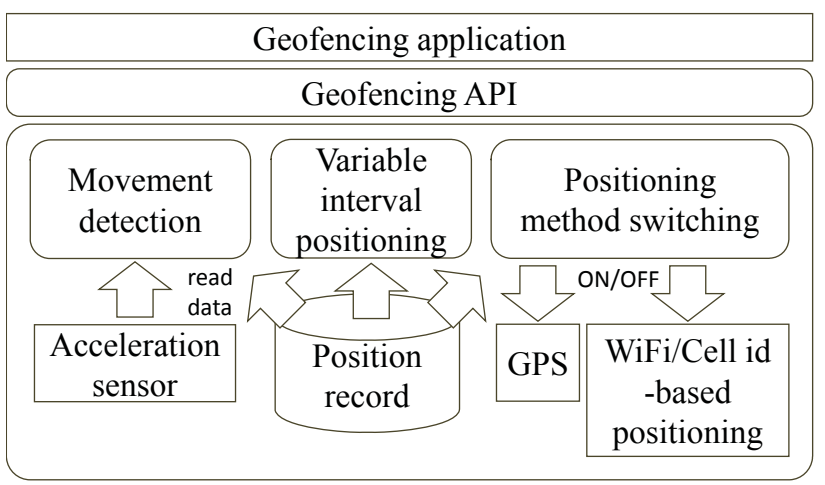

Figure.1. Sensor-fused energy-efficient geofencing system

\begin{tabular}{|l|l|l|}
\hline PROPOSED & Movement detection & $10 \mathrm{~min}$ \\
\cline { 2 - 3 } & $\begin{array}{l}\text { Assumed max } \\
\text { velocity }\end{array}$ & $100 \mathrm{~km} / \mathrm{h}$ \\
\cline { 2 - 3 } & $\begin{array}{l}\text { Positioning method } \\
\text { switching distance }\end{array}$ & $10 \mathrm{~km}$ \\
\hline $\begin{array}{l}\text { Android Geofence function } \\
\text { (PRIORITY_NO_POWER) }\end{array}$ & setFastestInterval() & $60 \mathrm{sec}$ \\
\cline { 2 - 3 } & setInterval() & $3,600 \mathrm{sec}$ \\
\hline
\end{tabular}

Table.1. Parameters for each method

Parameters utilized in the evaluation of Android Geofence function is shown in Table 1. In order to ensure detection delay to be less than 1 minute, fastest interval is set to $60 \mathrm{sec}$, which is the same condition as the proposed method. The parameter of setInterval (), which means desired interval, is set to 3,600 sec so that positioning interval is dynamically defined by variable interval positioning algorithm of Android Geofencing function.

\section{EVALUATION}

In order to initially evaluate the performance of the system, we utilized the following three scenarios that cover various situations of daily life: a stationary scenario, a moving scenario without entrance to the target spot, and a moving scenario with entrance. In the stationary scenario, the user stays at the starting point for five hours. In the moving scenario without entrance, the user keeps moving at the starting point for five hours without coming close to the target spot. In the last scenario, the user moves toward and enters into the target spot in two hours. The target spot is set to a station $50 \mathrm{~km}$ away from starting point in every scenario. Both of the proposed algorithm and Android Geofence function are implemented on Galaxy S III(Android 4.1.2).

From evaluation results of the stationary scenario and moving scenario without entrance, it is confirmed that the proposed method requires much less number of position fix compared to Android Geofence function. The number of position fix is shown as evaluation result, because it is an important factor to indicate battery consumption, and the battery consumption differs depending on the kind of devices. The result of the stationary scenario shows that even when the terminal is not moving, the Android Geofence (ST_NO_POWER) conducts position fix every five minutes, which is the same frequency as the moving scenario without entrance. On the contrary, the proposed method requires no position fix in the stationary scenario. In the moving scenario

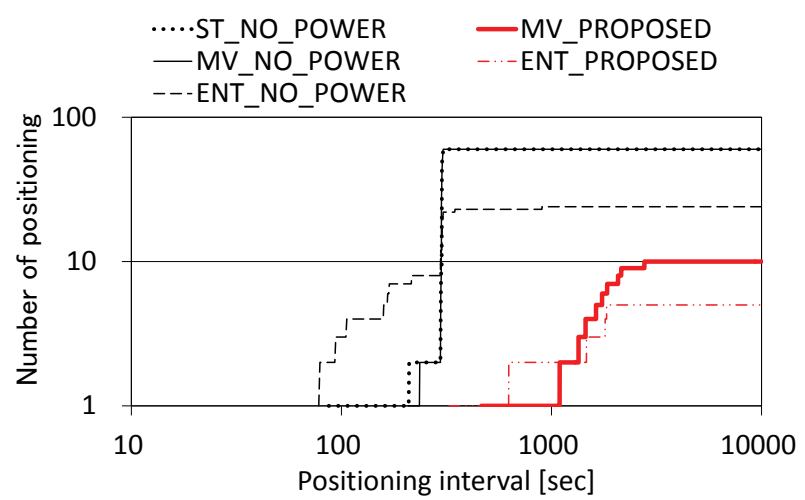

Figure 2. Cumulative frequency distribution of positioning

without entrance, the proposed method (MV_PROPOSED) decreases number of position fix by $83 \%$ compared to Android Geofence function (MV_NO_POWER). In the last scenario, it is confirmed that detection accuracy of the proposed algorithm is not degraded compared to Android Geofence function. Number of position fix is reduced by $79 \%$ in the proposed method (ENT_PROPOSED), while detection delay of both of the algorithms are 3 minutes. Though the degree of positioning count reduction will vary depending on evaluation scenario, This result shows that there is distinctive difference in variable interval positioning algorithms of each method.

\section{CONCLUSION}

In this paper we proposed sensor-fused energy-efficient geofencing system. Evaluation results with implemented system on Android-based smartphone shows that the proposed method decreases number of position fix by $79 \%$ compared to Android Geofence function, without any detection delay. In addition, it is clarified that the number of position fix is highly reduced in daily life especially when the terminal is staying away from the target spot. As a future work, we plan to analyze the effect of energy efficiency caused by each component of the geofencing system.

\section{REFERENCES}

[1] U. Bareth. Privacy-aware and Energy-efficient Geofencing through Reverse Cellular Positioning. In IEEE IWCMC 2012 Proceedings, pages 153-158, 2012.

[2] T. Farrell, et al. Energy-Efficient Monitoring of Mobile Objects with Uncertainty-Aware Tolerances. In IEEE IDEAS 2007 Proceedings, pages 129-140, 2007.

[3] J. Martens, et al. A declarative approach to a user-centric markup language for location-based services. In ACM Mobility Proceedings, 2009.

[4] T. Nakagawa, et al. Variable Interval Positioning Method for Smartphone-based Power-saving Geofencing. In IEEE PIMRC 2013 Proceedings, pages 3482-3486, 2013.

[5] C. Lee, et al. Energy-efficient Location Logging for Mobile Device. In IEEE SAINT 2010 Proceedings, pages 84-90, 2010.

[6] N. Deblauwe, et al. Hybrid GPS and GSM Localization Energy-efficient Detection of Spatial Triggers. In IEEE WPNC 2008 Proceedings, pages 181-189, 2008. 